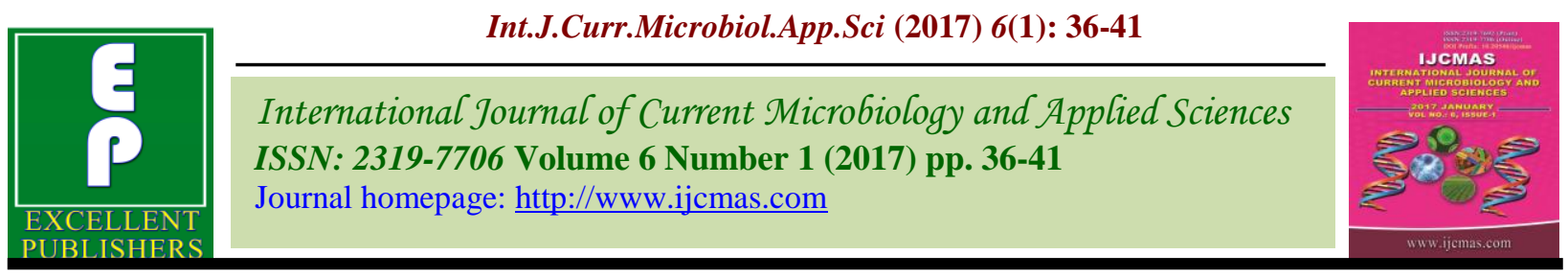

Original Research Article

http://dx.doi.org/10.20546/ijcmas.2017.601.005

\title{
Seroprevalence and Recent Trends of Dengue in a Rural Area in South India
}

\author{
Ruby Thomas* and K. Shilpa \\ Department of Microbiology, Akash Institute of Medical Sciences and Research Center \\ Prasannahalli road, Devanahalli, Bengaluru rural-562110, India \\ *Corresponding author
}

\begin{abstract}
A B S T R A C T
Keywords

Dengue,

Prevalence,

seasonal variation, seropositivity, thrombocytopaenia.

Article Info

\section{Accepted:}

09 December 2016

Available Online:

10 January 2017

Dengue is the most critical arthropod borne viral infection and a major public health concern globally in recent years infecting about 50 million people each year. Dengue infected patients present with symptoms varying from mild fever to life threatening shock. Associated thrombo cytopaenia is a hall mark of a life threatening dengue infection. A platelet count of less than $100,000 / \mu 1$ is a diagnostic criteria for dengue haemorrhagic fever (DHF) as per World Health Organization (WHO) guidelines, 2015. Thrombocytopaenia can be seen in both dengue fever (DF) and DHF. In this study, a total of 1228 patients both out patients and inpatients of Akash Hospital in Bengaluru rural were screened for dengue infection. A rapid-Immuno chromatographic test, (ICT) SD Bioline Dengue Duo ${ }^{\circledR}$ was used to run all the samples for detection of NS1antigen (nonstructural protein), IgM (immunoglobulin M) and IgG (immunoglobulin G). NS1 antigen is an early diagnostic marker, appears on the first day of fever. All positives were confirmed by using Bhat Bioscan ${ }^{\circledR}$ Dengue rapid test kit also detects NS1antigen, IgM and IgG. Out of 1228 samples 324 samples were seropositive. Platelet counts were performed on whole blood on these seropositive patients using Sysmex XS 800i automated haematology analyser. Among the 324 seropositive patients, 17(5.24\%) had severe thrombocytopaenia, 109 $(33.64 \%)$ were showing moderately low platelet count, 93(28.70\%) with a borderline count and $105(32.40 \%)$ had a normal platelet count.
\end{abstract}

\section{Introduction}

India has emerged as an endemic country harboring many mosquito borne diseases like malaria and dengue. There has been an increase in the incidence of dengue globally and over $40 \%$ of the world's population is now at a risk from dengue as per WHO 2016. There has also been an increase in the incidence of adult dengue in comparison to the children as reported in early years (Sarin et al., 1998). There is very limited data on adult dengue infections from South East Asia.
Dengue virus is a Flavivirus and has four distinct serotypes (DENV-1, DENV-2, DENV-3,DENV-4. Aedes aegypti and Aedes albopictus mosquitoes are the primary vectors. Recently in August 2016 Dr KK Aggarwal, General Secretary, Indian Medical Association was in Bengaluru to discuss dengue-prevention measures in which he stated that the research has now proved that the vectors of Dengue also bite during the night in areas where there is light. 
According to the U.S. Centers for Disease Control and Prevention (CDC), recovery from one serotype gives a lasting immunity from that serotype but subsequent infection with other serotypes increases the risk of developing severe dengue. There is no cross protection between the four serotypes but cross reactions can occur. The disease is noted to have a pattern of epidemic every 2-5 years (Swash, 1995).

Dengue is an acute febrile illness caused by any of the four types serotypes of the Dengue virus characterised by a spectrum of disease which includes biphasic fever, myalgia, retro orbital pain, arthralgia, rash and leukopenia. Dengue haemorrhagic fever is characterised by haemoconcentration, abnormality of haemostasis and in severe cases fluid and protein losing shock syndrome called Dengue Shock Syndrome (DSS).

According to the WHO criteria , for defining DHF the following must be present (a) fever (b) haemorrhagic tendency(c) thrombocytopaenia (d) evidence of plasma leakage, manifested by either a rise in haematocrit equal or greater than $20 \%$ above the average for age, sex and populations or signs of plasma leakage such as a pleural effusion, ascites and hypoprotenemia.

DSS has all the above mentioned criteria as in DHF plus evidence of circulatory failure manifested by rapid and weak pulse, narrow pulse pressure (less than $20 \mathrm{~mm} \mathrm{Hg}$ ), hypotension for age or cold, clammy skin.

In majority of patients, thrombocytopaenia is transient and asymptomatic, but significant numbers develop bleeding manifestations (Handian, 2001). Spontaneous bleeding is seen in patients who have a platelet count of $<$ $20,000 / \mu 1$ in most of the patients. Petichiae/purpura is seen in patients having a platelet count between 20,000 to $40,000 / \mu 1$.
This call for evaluating platelet count for all Dengue infected cases and follow up after platelet transfusion.

The main aim and objectives of this study to find out the prevalence of Dengue infections in this rural area of Bengaluru rural. And to find out the trend of seroprevalence in paediatric and adult dengue cases. Also to find out the incidence of thrombocytopaenia in these seropositive dengue patients. And to observe the months of outbreaks and its seasonal variation.

\section{Materials and Methods}

The present study was conducted between August 2015 and August 2016 at Akash hospital, Devanahalli, Bengaluru rural to observe the seroprevalence, seasonal variation of outbreaks and the incidence of thrombocytopaenia in seropositive dengue patients. Samples were obtained from 1228 suspected cases of dengue infection both from outpatients and inpatients. Serological diagnosis was done using the rapid ICT-SD Bioline Dengue Duo ${ }^{\circledR}$ which detects NS1,IgM and IgG. All positive samples were confirmed using another rapid ICT-Bhat Biotech ${ }^{\circledR}$ kit. The test can be performed using serum, plasma or whole blood.

The exclusion criteria were patients with thrombocytopaenia but serologically negative, patients with thrombocytopaenia and no fever and also cases if routine laboratory testing showed up as bacterial infections or any other viral infections other than dengue infection. The NS1 antigen is the first marker to be detected in the early stage of the disease even with a history of one day of fever and is present from 0 to 9 days after onset of symptoms, followed by $\operatorname{IgM}$ antibody which is detected from 5 to 10 days of primary infection and until 5 days of secondary infection. IgG antibodies appear after 14 days 
and persist for life in primary infection and rise within 1to 2 days after the onset of symptoms in secondary infection. It is important for the clinician to look for progression to DHF and DSS. Early detection of DSS is of utmost importance as it may lead to mortality if appropriate treatment is not administered at the earliest. This test is proved to be a simple, rapid and easy to perform in a rural set up for generating quick and reliable reports.

Platelet count was determined using Sysmex XS 800i automated haematology analyser using whole blood from dengue positive patients.

\section{Results and Discussion}

In this study, 1228 suspected dengue infected individuals were screened for dengue infection and 324 were seropositive (26.38\%).Among the positives 172 were males and 152 females as shown Fig 1. They were categorized into 3 age groups as follows 0 to 15 years had a total of 125 patients (71 males and 54 females).Whereas 183 patients were in the age group between 16 to 50 having 96 males and 87 females. Above 50 years there were 16 patients (5 males and 11 females). Male preponderance was observed in the paediatric age group as well as in the 16-50 years age group. There was female preponderance in the above 50 years age groups. Out of the 324 positives 230 were positive for NS1, 35 for IgM and 108 for IgG.. Out of the total positive cases both NS1 and $\operatorname{IgM}$ were positive in 34 patients, NS1 and $\mathrm{IgG}$ positive in 17 cases and $\mathrm{IgM}$ and $\mathrm{IgG}$ positive in 26 cases. Out of the 324 seropositive cases, $105(32.40 \%)$ had a normal platelet count.93 patients $(28.70 \%)$ showed up as borderline and $109(33.64 \%)$ as moderately low and 17 (5.24\%) showed as severely low count, as shown in Table1 and Fig 2.

There was a similar seasonal outbreak of dengue positive cases in both the years 2015 and 2016 in the monsoon period between the months of June to September as compared to the pre and post-monsoon periods as depicted in Fig 3.

According to some reports, Dengue has been reported from urban Bengaluru in the months of June to October when viral load is high and then the transmission falls in the remaining months of the year. Its now reported throughout the year as a result of environmental reasons like rise in construction activity, lack of clean water and sanitation. Increased disease transmission is observed when people live in close proximity in urban areas. International travel also helps in spreading the disease. In our study from the rural area between August 2015 and August 2016, there was a rise in dengue cases in the monsoon period (June to September) which indicates an active viral transmission during the monsoon period (Lall et al., 2016) and a lower prevalence in the rest of the months of the year.

The outbreaks coincided very well with the monsoon and post monsoon period. August 2015 showed out breaks of Dengue seropositivity of $39.43 \%$ which peaked in September 2015 (45\%) followed by a drop, $25.64 \%$ in November 2015. The other months showed decreased seropositivity. In the year 2016 there was an outbreak in the month of July with a seropositivity of $32.60 \%$ followed by a drop, $25 \%$ in the month of August 2016. 
Table.1 Association of Platelet counts with Gender and Age groups

\begin{tabular}{|l|l|l|l|l|l|l|}
\hline Characteristics & Group & \multicolumn{5}{|c|}{ Platelet count } \\
\cline { 3 - 7 } & & Normal (\%) & $\begin{array}{l}\text { Borderline } \\
(\%)\end{array}$ & $\begin{array}{l}\text { Moderately } \\
\text { low (\%) }\end{array}$ & $\begin{array}{l}\text { Severely } \\
\text { low (\%) }\end{array}$ & Total \\
\hline \multirow{3}{*}{ Gender } & Male & $55(52.38)$ & $47(50.53)$ & $60(55.04)$ & $10(58.82)$ & $\mathbf{1 7 2}$ \\
\cline { 2 - 7 } & Female & $50(47.61)$ & $46(49.46)$ & $49(44.95)$ & $7(41.17)$ & $\mathbf{1 5 2}$ \\
\hline \multirow{3}{*}{ sge } & $<=15$ & $31(29.52)$ & $39(41.93)$ & $44(40.36)$ & $11(64.70)$ & $\mathbf{1 2 5}$ \\
\cline { 2 - 7 } & $16-50$ & $66(62.85)$ & $50(53.76)$ & $62(56.88)$ & $5(29.41)$ & $\mathbf{1 8 3}$ \\
\cline { 2 - 7 } & $>50$ & $8(7.61)$ & $4(4.30)$ & $3(2.75)$ & $1(5.88)$ & $\mathbf{1 6}$ \\
\hline Total & $\mathbf{n}(\boldsymbol{\%})$ & $\mathbf{1 0 5}(\mathbf{3 2 . 4 0})$ & $\mathbf{9 3}(\mathbf{2 8 . 7 0})$ & $\mathbf{1 0 9}(\mathbf{3 3 . 6 4})$ & $\mathbf{1 7}(\mathbf{5 . 2 4})$ & $\mathbf{3 2 4}$ \\
\hline
\end{tabular}

Fig.1 Age group of Patients

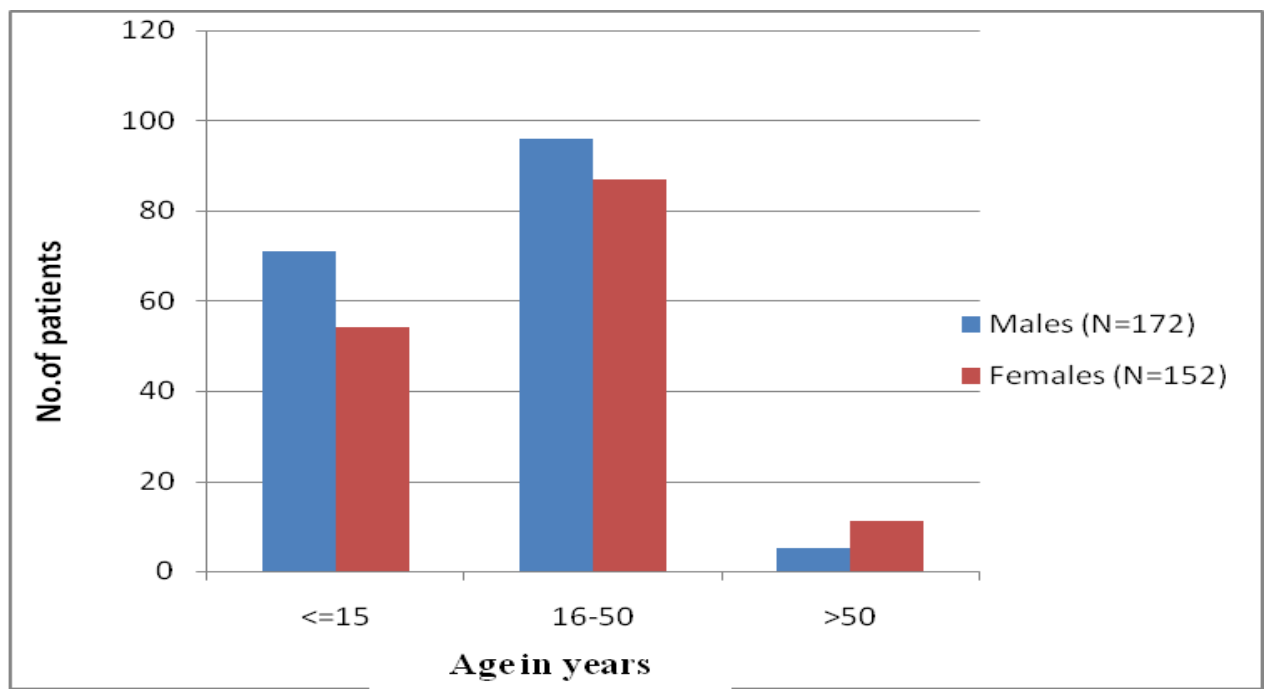

Fig.2 Association of Platelet counts with Age groups

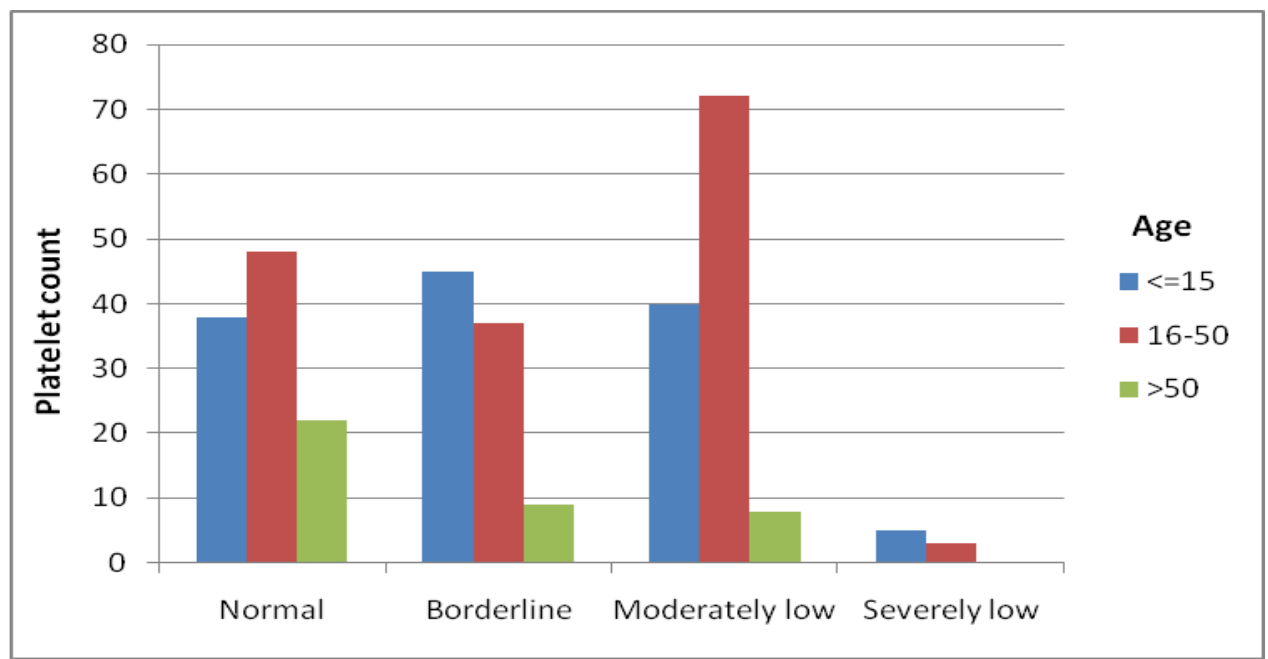


Fig.3 Month wise distribution of total number of suspected cases and serologically dengue positive cases

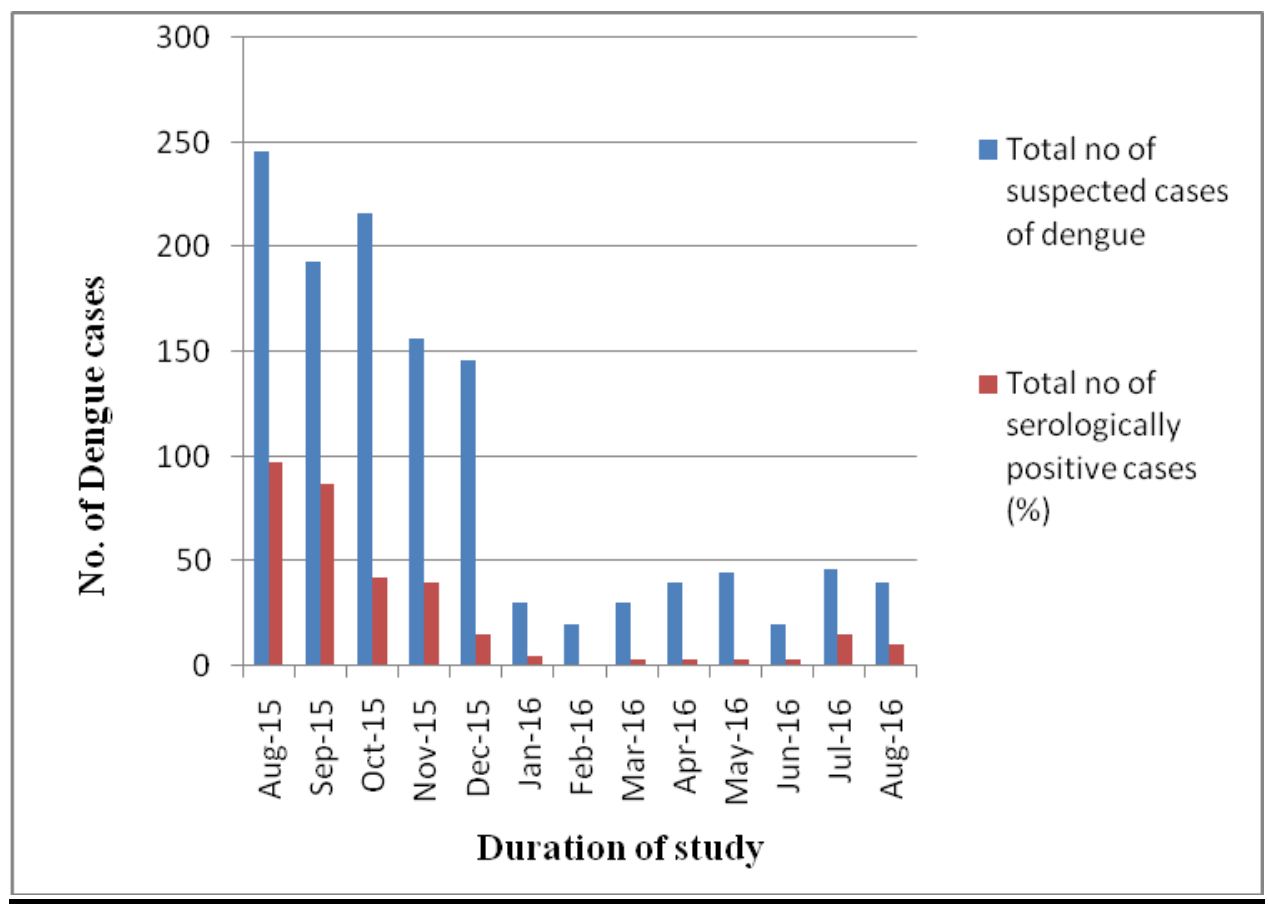

Also there were insignificant numbers of seropositivity between the months from January to June. These figures shows that there was an increase in the seropositivity in the monsoon period between the months June to September in comparison to the post monsoon period, a similar observation was made in both the years. This was is in contrast to a study done by (Deepthi et al., 2012). Dengue affects both children and adults in and around Devanahalli (Bangalore Rural) but in our study adult dengue cases (61.41\%) were more compared to dengue cases in paediatric age group (38.58\%).

Dengue is one of the re-emerging viral infections. India is now being affected with several outbreaks every year, increase in the number of cases reported and also showing an increase in the severity of the disease

As there is no specific drug available and since the vaccine is still in the developmental stage, treatment is still only supportive
.Therefore the control measures should be aimed at the vector control. There is an urgent need to strengthen national programmes for preventing Dengue and DHF. This will also help us to control other mosquito borne diseases like malaria, chikungunya, Japanese encephalitis and filaria in India.

The laboratory surveillance also has to be enhanced in different parts of the country for prompt and early diagnosis. The state should pave the way for evolving effective vector control strategies both in rural and urban areas to curb this growing disease.

\section{References}

Deepti, P., Shashikala, P. and Vasavi Shenoy. 2012. Evaluation of platelet count in Dengue Fever Along with Seasonal Variation of Dengue Infection. J. Blood Disorders Transf., 3(4):1-4.

Halstead, S.B. 2007. Dengue. Lancet, 370: 1644-1652. 
Handian, R.L. 2001. Bleeding and thrombosis. Harrison principles of internal medicine (15 th edn). McGraw Hill, USA.

Kroger, A., Nathan, M., and Hombach, J. 2004. World Health Organization TDR Reference Group on Dengue. Dengue. Nat. Rev. Microbiol., 2: 360-361.

Lall, H. 2016. Sero-Prevalance of Dengue in Tertiary Care Hospital in Delhi. Int. J. Curr. Microbiol. Appl. Sci., 5(6): 439445.

Nimmannitya, S. 1987. Clinical spectrum and management of dengue haemorrhagic fever. Southeast Asian J. Trop. Med. Pub. Hlth., 18(3): 392-397.

Sarin, Y.K., Singh, S. and Singh, T. 1998. Dengue viral infection. Indian Pediatr., 35: 129-137.

Swash, M. 1995. Doctor and patient. Hutchison's Clinical Methods $20^{\text {th }}$ edn.

Teixeira, M.G., Barreto, M.L. 2009. Diagnosis and management of dengue. BMJ, 339: b4338.

WHO. 2011. Comprehensive Guidelines for prevention and control of Dengue and Dengue Hemorrhagic Fever. Revised and Expanded Edition.

\section{How to cite this article:}

Ruby Thomas and Shilpa, K. 2017. Seroprevalence and Recent Trends of Dengue in a Rural Area in South India. Int.J.Curr.Microbiol.App.Sci. 6(1): 36-41. doi: http://dx.doi.org/10.20546/ijcmas.2017.601.005 